\title{
New Approach for Laser Dental Education
}

\section{J.Kameenoff}

Prof. Dr Julia E. Kamenoff, Doctor, DDS, Ph.D., HDG, President BALDM, Medical University, Faculty of Dental Medicine, Deptm Prosthodontics, President of Career Center - Laser Center for postgraduate training, Sofia, Bulgaria

*Corresponding author: Prof. Dr Julia E. Kamenoff, Doctor, Medical University, Faculty of Dental Medicine, Deptm Prosthodontics, 1-3, "GeorgiSofiiski”blv, Faculty of Dental Medicine, Pr.off 810, fl.8, Sofia 1440, Bulgaria; E-mail: baldm.fdm@gmail. com

Received Date: January 20, 2019; Accepted Date: February 13, 2019; Published Date: February 18, 2019

Citation: J.Kameenoff (2019) New Approach for Laser Dental Education. J Dent Oral Health 6: 1-6.

\begin{abstract}
Purpose: The author has presented a new approach for Laser Dental Education based on Biological Medicine principles. The new educational program involves knowledge about laser biostimulation, photo infrared photo biomodulation, Laser Eliminating Therapy as Allergy and Environmental Medicine, Immunological Allergies, Autonomic nervous System Hypersensitivity Allergy and alternative methods of Combined Laser Treatment. This new approach of teaching show how to build Model of Individual Optimum Laser Stimulation and require education on four levels: First level - laser biostimulation and biomodulation, Second level - Healing effect of Infrared Laser beam, clinical application. Next third level involves skills on Laser acupuncture and Combined Laser Biosynergetics. The higher level of laser education gives information about Turbulence Laser Medicine clinical application. All levels of Laser training require workshops with different types of Dental laser machines. This new approach of education has been approbated in the Department of Prosthodontics, Laser Educational center for post graduated education at the Faculty of Dental Medicine, Medical University, Sofia. Results after the laser education show great effectiveness.
\end{abstract}

Keywords: Laser Dental Education, Curriculum guidelines for laser education

\section{Introduction}

The Career center - Laser center for postgraduate education at the Faculty of Dental Medicine-Sofia was established as a university unit to support communication, good work and mutual benefit between dental practices, academia, and students. The main focus of the team's work is the provision of high-quality service in the partnership with employers in the field of dental practices, as well as the development of programs, strategies and practical seminars for the professional and career development of students.

\section{Main body}

Laser medicine develops very quickly and is widely used in everyday Dental practice. In recent years the use of lasers has expanded almost in all areas of Dental Medicine thanks to the integration of scientists with different specialties (physicists, chemists, opticians' engineers, biologists, biochemists, etc.) from all over the world. Worldwide Laser clinical institutes and academies have been established, which

(C)2019 The Authors. Published by the JScholar under the terms of the Creative Commons Attribution License http://creativecommons.org/licenses/by/3.0/, which loize.marechal@umontreal.ca

maximilien.laviolette-brassard@umontreal.ca have brought together their specialists in global scientific and clinical research in order to create unified and efficient use of the diverse laser apparatus and systems that World markets [8-11]. The different lasers are distinguished by their own physical parameters and in practice, it is very difficult to standardize and randomized Laser therapy (LT) $[1,6,7]$. Bulgaria is actively involved in such developments. Prerequisite for this is the development of Low and High energy Laser Treatment (PDT and HELT) in Bulgaria and the production of Bulgarian diode laser systems. The rapid development of laser therapy in recent years has led to an increase in quality requirements for laser-assisted therapy. New analytical methods are continually being developed, theoretical and virtual models and techniques are being developed this is the reason for changing the manner of laser training and for creating a new approach for laser Dental training [2-7]. Based on the knowledge about mechanisms of laser beam action and laser bioenergetics as Grothus and Soliton mechanisms, and Cable Hypothesis, our educational program has been developed. The most important postulate in our point of view is building of the individual Model of optimum Laser Stimulation after the special functional diagnostic process and clinical assessment of four Criteria -bio psychological patient model, therapeutic program, functional anatomy estimation, and etiologic factors Figure.1. 


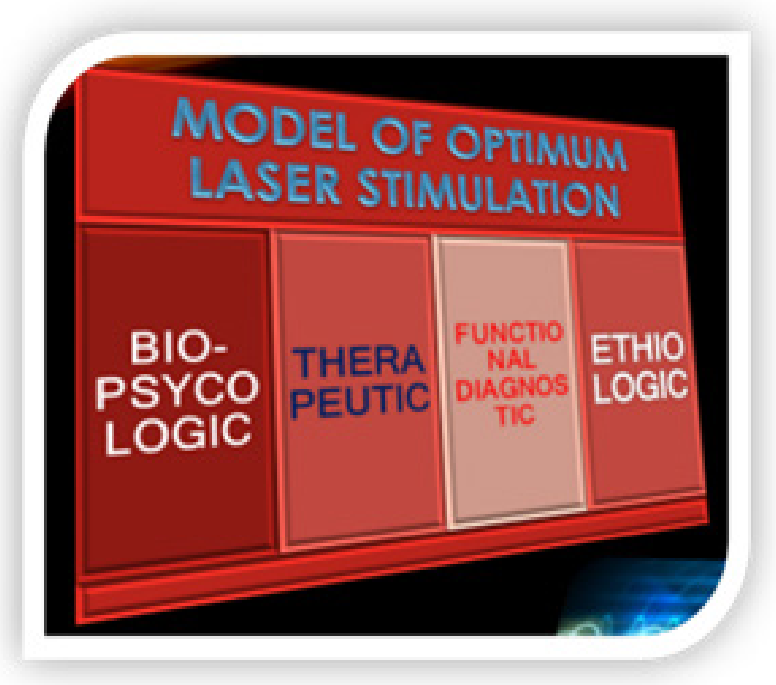

Figure.1 Model of Optimum laser stimulation involving four models of the patient healthy condition individually.

The main problem in education on oral laser application on a global scale to date is the lack of a sophisticated methodology that allows a concrete visualization of the results obtained. In recent years, there has been a significant increase in the interest in proving the healing effect of PDT and HELT [9]. The vagueness of the mechanism of interaction of light photons at all levels of the human body's own biological organization gives rise to uncertainty in laser therapy practitioners. The main problem is the calculation of laser power and energy [8]. An objective, randomized system for controlling the curative effect has not yet been established on clinical criteria confirming the proper course of laser bioenergy and bio-synergy. The question of the healing power of the laser apparatus is not resolved. No in-depth standardized study on the loss of laser radiation in laser machines with a conductive fiber optic system such as diode lasers has been done Therefore, a second major postulate in our program is the dependence of the optimal dose of laser radiation on irradiation approach, emit ion mode and exposure time. The training in our laser center is carried out with a lot of prepared patients protocols and methods for building individual therapeutic programs depending on the compensatory possibilities of the body of patients from different compensatory models of clinical situations Figure.2.

World literature provides information on scientific controversies and fluctuations at the recommended optimal dose of electromagnetic radiation on the human body $[1,8]$. It has been shown that tissue reactions are variable in nature under the influence of different physiological internal and external states in the dynamic self-regulating system of the human organism [3-6]. The current nature of the problem is determined by the direct relationship between therapeutic efficacy and biological factors such as total body reactivity, regenerative and reprocessing processes, oxidative and reductive potentials, regional and general blood flow, the presence of pathogenic associations of microorganisms and the neuromuscular regulation of the function $[2,7]$.

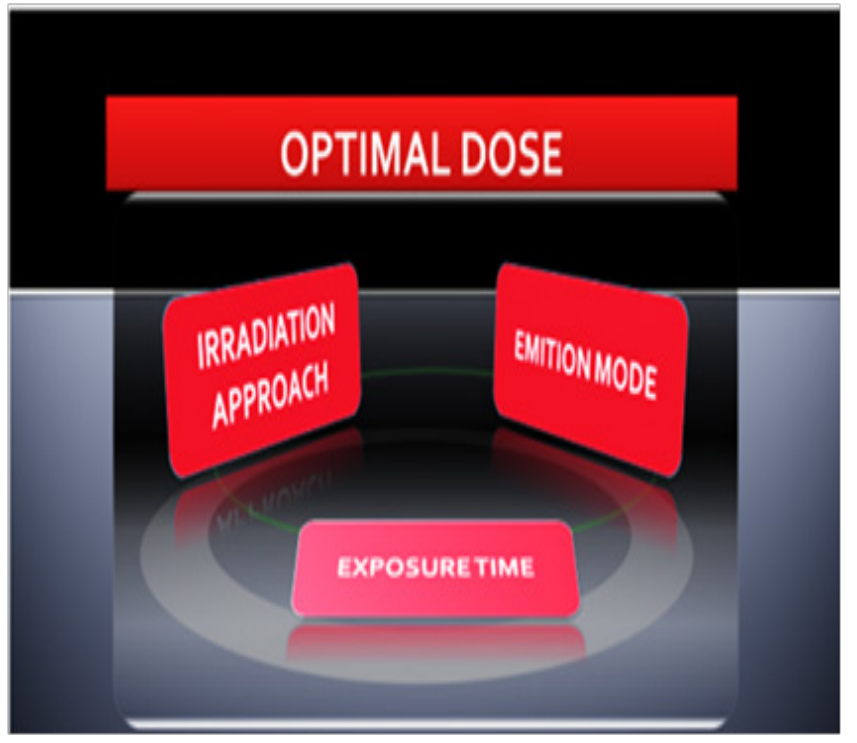

Figure.2 Schematic illustration of the methodical approach for choosing the optimal dose of laser radiation individually for each patient and for each stage of treatment

Particularly relevant is the topic of laser energy therapy with coherent monochromatic laser radiation and programmable output power in the mean range of infrared electromagnetic radiation $[7,8]$. A key clinical parameter for assessing therapeutic efficacy is the amount and density of the applied laser light power [8]. The next goal is to master new frequency bands, which is related to the development of new measurement methods. Particularly relevant is the topic of the resonant phenomenon of tissue response under the influence of electromagnetic radiation combining frequencies with a specific therapeutic effect. A qualitatively different approach is needed to confirm the healing effect of known frequencies and to recommend new frequency ranges. The scientific research in the field of medical knowledge of the physical parameters of the laser devices is the most recent one for their flexible combination for optimal dosing of the working power and energy and precise dosing of their density - optimal clinical dose of laser therapy.

Laser Therapy Standardization - many prominent scientists and experienced clinicians have established "Golden Standards" to treat dental pathology in all its varieties [8]. Unfortunately, the differences in laser performance and the theoretical rationale of the authors do not explicitly confirm the effectiveness of laser therapy. Laser dentistry is distinguished by experimental and clinical studies not only on pathological processes but also on new diagnostic methods, prophylactic approaches and treatment strategy [1-9]. Therefore, a number of issues remain unresolved. New profiles in laser medicine such as Laser Photodynamic Therapy, Laser Biological Medicine, Laser Bioenergy and Bio synergy, Combined and Hybrid Laser Therapy, Quantum Medicine and Resonance Frequency Therapy. That is why, in the Faculty of Dentistry at the Medical University in Sofia, a laser center for postgraduate training in laser dentistry was established, in which the teaching is in harmony with all the world educational standards and 
programs and the lecturers are habilitated lecturers with high qualification and worldwide certification. Their knowledge has been verified at certification exams and their skills to work with different types of laser systems have been proven. Some of the lecturers at the laser center are lecturers at the European University for Postgraduate Dental Laser Practice (EMDOLA - European Master Degree of Oral Laser Application). Currently, the laser dental center at the Faculty of Dental Medicine in Sofia is developing three large-scale scientific projects and, with a very modern technique, conducts in-depth clinical studies on the healing effect of the most commonly used laser systems in our country. The clinical and experimental work of the Center is entirely focused on building modern therapeutic paradigms and creating new standardized therapeutic methods. A serious university academy for laser therapy training has been set up in Bulgaria with a membership of 22 young and ambitious teachers - associated professors, Ph.D., students and associate students who have wonderful prospects for development in both research and clinical practice. New profiles in laser medicine are being developed, such as laser biology and laser bio synergy. The "usability" of these areas in the country could be a prerequisite for building a structure for controlling laser-assisted treatment by building a network infrastructure to address these and other novelties in electromagnetic medicine. The Center was established in order to provide a fundamental, clinical and practical situation for the establishment of a system of standards tailored to the individual optical characteristics and in compliance with the worldwide standards, such as the Curriculum guidelines for Laser Education, ALD, USA.

When Bulgarian Association of Laser Dental Medicine (BALDM) was founded in 2005 at the Faculty of Dental Medicine of the Medical University in Sofia, with Prof. Julia Kamenoff, under the auspices of the European Association of Laser Therapy Center in Vienna (ESOLA, Austria) under the leadership of Prof. Andreas Moritz,we created our own Educational program; characterized by followed special features

1. Objectives The objectives of the residency program are to:

- $\quad$ Basic knowledge on Laser physics, Laser-Tissue Interaction and Laser Safety according to Curriculum Guidelines and Standards - ALD

- $\quad$ Clinical experiences to ensure proficiency in all aspects of Laser Medicine, Biological Medicine and Turbulence Medicine

- $\quad$ Clinical experiences to ensure proficiency in diagnosis, treatment planning, and management of multi-disciplinary cases.

2. Provide biologically and scientifically based education in order to:

- Develop, monitor, and update all prosthodontic seminars and conferences to present contemporary, technologically and biologically-oriented information;

- $\quad$ Provide opportunities for scholarly activities, applied research, scientific articles, and clinical and or research presentations.

3. Prepare and train residents for a career in Laser Dental practice and/or academics to:

- require all residents to complete annual comprehensive examinations

- provide opportunities for completion of a 4 treatment case for every level of training

- provide residents with opportunities to pursue an academic degree(s) in related disciplines: Laser Cavity Preparation, Laser Endodontics, Laser Periodontology, Laser Assisted Prosthodontics, and Implantology, Laser Assisted Child Dentistry and Orthodontics, Laser Surgery

4. $\quad$ Provide quality and professional care to all patients to:

- $\quad$ Ensure ethical and professional conduct by all individuals involved in patient care;

- $\quad$ Ensure proper care of all patients in the Laser Medicine residency program;

- $\quad$ Ensure patient satisfaction with the care they receive.

5. Residency Education - The Faculty of Dental Medicine offers Laser Advanced Dental Education and Residency Programs leading to a Certificate of Completion in the respective program. Programs of four-year duration offer an optional educational tract leading to a Master of Dental Science degree (MDS) on Oral Laser Application.

6. About the program: Curriculum in four levels education:

- $\quad$ First level - the same as Standard Proficiency level ALD, but only about Photo Dynamic Therapy (PDT) - red visible light : laser physics, the nature of red laser beam, tips of Dental lasers, emitted in red visible light, laser-tissue interaction and healing effect of red diode lasers -intraoraly, extraoraly, laser magnet treatment, redsuperficial laser acupuncture in Dentistry, practical work on patients - for 1 week .

- $\quad$ Second level - Infrared laser beam and healing effects of different wavelengths in near, mid ane far infrared electromagnetic spectrum, infrared PDT methods, high-level lasers clinical approaches. Combined laser treatment - red and infrared different wavelengths by diode PDT (intraoral techniques, extraoral methods, deep laser acupuncture for pain relief, laser magnet treatment, trigger point therapy, laser antioxid therapy, elimination, and allergy treatment)Practice work every day -1 week.

- $\quad$ Third step - Laser acupuncture, photo infrared photobiomodulation, Dao in laser tonification, laser-assisted prosthodontics, periodontics, endodontics and sedation therapy, massages and body passages, laser-assisted aesthetic Dentistry and cosmetic procedures. Practice work every day.

- $\quad$ Final step - laser biosinergetics - combined methods of treatment with LLLT and High power level lasers - LLLT diode machines, RJlasers, Biolase, Doctor Smile etc. Practice work every day and a possibility for master degree in Medical University, Faculty of Dental medicine - Sofia, Bulgaria

7. Turbulence Laser Medicine education: our New approach for laser education is fully completely described in book "Laser Diode Systems Application in Dental Medicine" Figure.3 


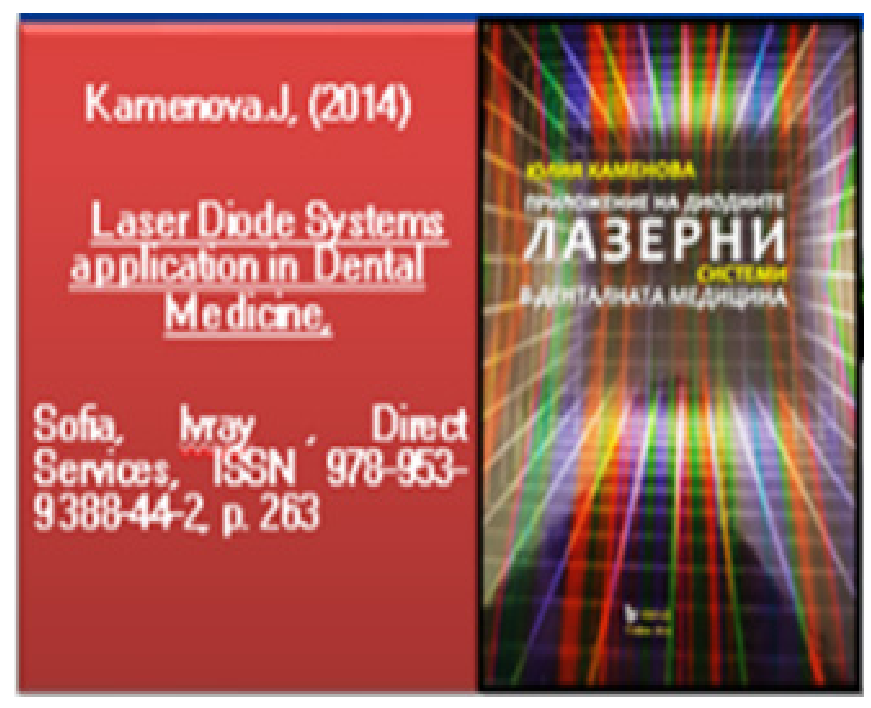

Figure.3 "Laser Diode Systems Application in Dental Medicine”, Kamenova. J, 2014, IVRAY, Direct Cervices, ISSN 978953-9388-44-2, Sofia, Bulgaria

Turbulence Laser Medicine Training with patients.

\section{Clinical procedures}

1. Red Laser Therapy - PDT, Photobiomodulation

- Red Laser Photobiostimulation: inflammatory acute/ chronic state: ulcers, wounds, mucosal lesions, soft tissue regeneration

Red Laser Photobiostimulation: sedation, TMJ sedation, intraoral sedation, Trigger point sedation

- $\quad$ Red Laser Photobiostimulation: anesthesia

- $\quad$ Red Laser Acupuncture: face sedative acupuncture, body sedative acupuncture, analgesic body acupuncture sedation

- $\quad$ Laser Magnet Therapy

- Red Laser Acupuncture, Laser Magnet Therapy,

TENS relaxation

Red Laser nonthermal photodynamic neuromuscular

\section{IR Laser Therapy - PDT, Photobiomodula-} tion, PIPBM

- IR Laser Photobiomodulation - tonification

- IR Laser Photobiomodulation - deep sedation

- IR Laser Photobiomodulation - TMJ tonification

- $\quad$ IR Laser Photobiomodulation - intraoral tonification

- $\quad$ IR Laser Photobiomodulation - Trigger zone therapy

- IR Laser Photobiomodulation - face tonification and cosmetics

- IR Laser Photobiomodulation - body tonification and analgesic body tonification

- IR Laser Photobiomodulation - Laser Magnet Thera-

py

- $\quad$ IR Laser Photobiomodulation - thermal PIPBM
3. Pain relief

- $\quad$ Combined Laser Therapy

- $\quad$ Extraoral Laser Magnet Therapy

- Extraoral Laser Magnet Therapy, Laser acupuncture,

TENS

- $\quad$ Pain relief in redness, heat and swelling acute inflammatory stage

- Pain relief in less of function: chronic inflammatory stage

- Pain relief in surgery: post extraction rehabilitation

- $\quad$ Pain relief in Dental, Myofascial, muscle, soft and hard tissues symptoms

- Painless teeth preparation

4. Modern Biological Medicine

- $\quad$ Laser Advanced Biosynergetics

- Laser Energetics in synergic action

- $\quad$ Laser Energetics in muscles

- Laser Energetics in bone structures

- Laser Synergetics in Oro - Facial complex

- $\quad$ Laser Energetics and Biosynergetics in soft and hard tissue support

\section{Hard Tissue Procedures}

- Caries removal and cavity preparation

- Laser-assisted etching

- Osseous crown lengthening, osseous contouring

6. Soft Tissue procedures

- $\quad$ Laser Assisted Prevention and Cleaning

- Laser Assisted Gingival recontouring: gingivoplasty, gingivectomy, gingival toughing for crown impressions, gingival sculpting

- Implant exposure

- $\quad$ Frenectomy

- $\quad$ Benign and malignant lesion removal

- Operculectomy

- $\quad$ Gingival hypertrophy reduction

- $\quad$ Soft tissue crown lengthening

- $\quad$ Preprosthetic surgery

- Excisions and odontectomy

- Incisions and drainage of abscesses

7. Endodontic Laser Treatment

- Tooth preparation to obtain root canal

- Pulpotomy

- Pulp extirpation

- $\quad$ Laser pain relief in pulp hypersensitivity

- Root canal cleaning

- Root canal decontamination

- Pulp cavity hemostasis

- $\quad$ Pain reduction after endo - treatment

- Laser acupuncture anesthesia in endodontics

\section{Laser Assisted Periodontal Treatment}

- Tartar deposits cleaning

- $\quad$ Laser Assisted Oral Hygiene 
- Sulcular debridement

- Pocket Sterilization

- LANAP

- Intrasulcular excision

- Granulation tissue removal

- Bone Laser Ablation

- $\quad$ Perio - and bone laser surgery

- Informative Laser Frequency - Resonance IR Laser Periodontal Therapy

9. Turbulent Laser Medicine - TLM

- TLM in systematic effect on peripheral NS

- TLM in systematic effect on CNS, Trigeminal Neural-

gia

- TLM in Occlusal Trauma

- TLM in Malocclusions

- $\quad$ TLM in Myofascial disorders

- TLM in Occlusal Adjustment

- $\quad$ TLM in Full Mouth Restoration and Prosthetic Rehabilitation

- $\quad$ TLM in TMJD

- TLM in Osteolysis and progressive bone loss

- $\quad$ TLM in Teeth Mobility

- $\quad$ TLM in orofacial pain, neck pain, low back pain, TMJ

Disorders, Myofascial Dysfunctional Pain Syndrome, Costen Syndrome

- $\quad$ TLM in Occlusal and Facial support in splint Therapy or Maxillofacial Prosthodontics

- TLM in periodontal disorders: laser soft tissue decontamination, bleeding reduction, furcations, cervical recessions, increasing probing depth, gingival crevice, deep abnormal pockets formations, epithelial attachments improvement, teeth migrations, bone loss, acute - chronic periodontitis, for bacterial population reducing.

- TLM in Orthodontics: vertical and horizontal dimensions improvement, occlusal vertical and horizontal dimensions restoration

- $\quad$ TLM in destroyed overall Dental Appearance - Full Mouth Prosthetic and Orthodontic Restoration

- TLM in Occlusal Aesthetic Restoration and Cosmet-

ics

- TLM in dental arches correction and rehabilitation

- TLM in horizontal growth osteosynthesis.

- TLM in Esthetic Dentistry

During the followed stage of own TLM - education, clinical cases, grouped by the characteristic of each model clinical traits are presented and discussed. For each compensatory model of the clinical situation modeling, we are proposing an algorithm for successful treatment.

Our new Graduate Program offers basic, translational, and clinical studies in oral pathology, biomedical research, and health that span spatial scales from the Allergology and Environmental Medicine

\section{Conclusion}

- $\quad$ All educational programs at our Laser center for postgraduate education are designed to train the dental resident, in the most comprehensive manner, to use different laser machines.

- $\quad$ All levels of Laser training require workshops with different types of Dental laser machines. This new approach of education is approbated and developed in Department of Prosthodontics, Laser Educational center for post graduated education at Faculty of Dental Medicine, Medical University, Sofia, Bulgaria. Results after the laser education show great effectiveness. 


\section{References}

1. Niemz MH (2007) Laser-tissue interaction. Fundamentals and applications. 3rd enlarged ed. Berlin, Germany: Springer 45-46.

2. Russel AD (2003) Lethal effects of heat on bacterial physiology and structure. SciProg 86:115-137.

3. Knappe V, Frank F, Rohde E (2004) Principles of lasers and biophotonic effects. Photomed Laser Surg 22:411-417.

4. Joffe SN (2003) Lasers in medicine. In: Driggers RG, editor. Encyclopedia of optical engineering, volume 2. New York: Marcel Dekker, Inc., 1045-1056.

5. Springer TA, Welch AJ (1993) Temperature control during tissue welding. Appl Optics 32:517-525.

6. McKenzie AL (1990) Physics of thermal processes in laser-tissue interaction. Phys Med Biol 35:1175-1209.

7. Miserendino LJ, Levy G, Miserendino CA (1995) Laser interaction with biologic tissues. Chapter 3 in Miserendino LJ, Pick RM, editors. Lasers in dentistry. Chicago: Quintessence Publishing Co., Inc., 39-55.

8. Coluzzi DJ (2008) Fundamentals of Lasers in Dentistry. J Laser Dent 16:4-10.

9. Seka W, Rechmann P, Featherstone JDB, Fried D (2007) Laser ablation of dental hard tissue. J Laser Dent 15:6172.

10. Parker S (2007) The use of laser energy for therapeutic ablation of intraoral hard tissues. Position paper: Science and Research Committee, Academy of Laser Dentistry. J Laser Dent 15:78-86.

11. Sweeney C (2009) Laser safety in dentistry: a position paper. The laser safety committee, Academy of Laser Dentistry. J Laser Dent 17:39-49.

\footnotetext{
Submit your manuscript to a JScholar journal and benefit from:

๑ Convenient online submission

ฯ Rigorous peer review

- Immediate publication on acceptance

ब Open access: articles freely available online

ब High visibility within the field

9 Better discount for your subsequent articles

Submit your manuscript at

http://www.jscholaronline.org/submit-manuscript.php
} 